\title{
Remarques sur la biotopologie, la morphologie, la cytologie et la taxinomie du Sporotrichum schenckii (Hetkoen et Perkins 1900)
} par Monique THIBAUT

Laboratoire de Parasitologie et de Mycologie ( $\mathrm{P}^{\mathrm{r}} \mathrm{M}$. LARIVIÈRE) U.E.R. Cordeliers, 15, rue de l'Ecole de Médecine, F 75006 Paris

\section{Résumé}

Le Sporotrichum schenckii (Hetkoen et Perkins, 1900), champignon pathogène, peut mener une vie saprophytique indépendante dans l'environnement de l'homme. La sporotrichose est une maladie des sujets manipulant la terre et surtout les végétaux.

Microscopiquement, les hyphes délicates, ramifiées et septées portent des conidies latéralement ou en bouquets terminaux sur les rameaux latéraux.

L'ultrastructure de la phase mycélienne a été étudiée au microscope électronique. La paroi cellulaire et le septum sont formés de plusieurs couches distinctes. Ce champignon semble caractérisé par la présence d’un « appareil synaptique ». Quelquesuns de ces caractères ressemblent à certains de ceux des algues. Des corps de Woronin sont associés intimement avec les septa.

La morphologie de la phase tissulaire du Sporotrichum schenckii chez l'hôte animal a été étudiée. Cet organisme est habituellement décrit dans les tissus sous la forme en cigare, mais l'examen des préparations de matériel expérimental montre son polymorphisme.

Sur milieu au sang ou sur milieu liquide de Mariat à $37^{\circ}$, les cellules de la phase levure sont soit isolées, soit bourgeonnantes. Elles sont très polymorphes.

L'ultrastructure de la phase levure est par beaucoup de points 
semblable à celle des levures étudiées par d'autres auteurs. Mais la paroi cellulaire est en général sensiblement plus épaisse dans la forme levure que dans la forme mycélienne et les effets de l'anaérobiose sur la structure mitochondriale sont évidents.

Le stade parfait du Sporotrichum schenckii a été découvert. Le nom de Dolichoascus schenckii Thibaut et Ansel, 1970 est proposé pour ce stade parfait, qui constitue un nouveau genre d'Endomycetaceae.

\section{Summary}

Sporotrichum schenckii (Hetkoen and Perkins 1900), pathogenic fungus, may have an independant saprophytic existence in man's environement. Sporotrichosis also occurs in persons exposed to soil and above all to plants.

Microscopically, the delicate, branching, septate hyphae bear conidia laterally or in groups from the ends of lateral branches.

The ultrastructure of the mycelial phase has been studied by electron microscopy. The cell wall and the septum consist of several distinct layers. This fungus appears to be characterized by possessing a «synapsis apparatus». Some of these characters resemble those of some algae. Intimately with all the septa are Woronin bodies.

The morphology of the tissue phases of Sporotrichum schenckii in the animal host has been studied. This organism seen in tissue is usually described as cigar shaped, but a review of preparations of experimental material indicated the polymorphism.

On blood agar or on Mariat's infusion at $37^{\circ}$ the yeast like cells appear as single or budding cells. They are quite polymorphic.

The fine structure of the yeast like cells was in many ways similar to that of yeasts studied by other authors. But, the cell wall was found in general slightly thicker in the yeast like cells than in the mycelial phase and the effect of anaerobiosis on mitochondrial structure was evident.

The perfect state of Sporotrichum schenckii was discovered. The name of Dolichoascus schenckii Thibaut and Ansel 1970 is proposed for this perfect state, which proved to be of a representative of a new genus of the Endomycetaceae.

Au cours de notre étude du Sporotrichum schenckii (Hetkoen et Perkins 1900), nous avons rencontré un certain nombre de difficultés, dues :

— à la très petite taille du champignon, voisine de celle des bactéries ;

- à des phénomènes complexes, mis en jeu pour obtenir la transformation de la phase mycélienne en phase levure; 
- à la mauvaise pénétration des fixateurs et des colorants dans un mycélium à consistance caoutchouteuse ;

- à la richesse en substances mucilagineuses des parois de la forme levure.

L'intérêt de nos observations sur le Sporotrichum schenckii provient, d'une part, de son mode de vie dimorphique, le champignon étant à la fois un parasite facultatif et accidentel de l'homme ou de certains animaux, et normalement un saprophyte vivant sur des plantes mortes; d'autre part, de l'importance des végétaux comme facteurs de son inoculation à l'homme et aux animaux. Les plantes en jeu peuvent être aussi bien des Cryptogames (mousses et en particulier sphaignes; Prêles), que des Phanérogames (fragments de tiges pourries d'œillets, épines de Berberis, de rosiers, d'AEchmea, écorce de hêtre, bois d'Acacia mollissima, grains d'Avena sativa). Le rôle des végétaux et du sol dans la transmission de la maladie est beaucoup plus important que celui des animaux.

La transformation de la phase mycélienne en une forme levure, qui est semblable à l'état parasitaire du champignon, ne peut se faire in vitro que dans des conditions culturales bien définies et complexes: le milieu liquide, de $\mathrm{pH} 7,2$, doit contenir des acides aminés (en particulier l'arginine, l'alanine et le glycocolle), des vitamines comme la thiamine, qui est indispensable, et de plus des oligoéléments. L'atmosphère du milieu doit être riche en anhydride carbonique. Enfin, la culture doit être placée dans une étuve à $37^{\circ}$ et agitée continuellement dans les trois dimensions.

\section{I. - Résultats apportés par nos recherches morphologiques et ultra-structu- rales sur la phase mycélienne du Sporotrichum schenckii.}

A) Microscopie photonique.

Notre étude en microscopie optique nous a permis de constater que le Sporotrichum schenckii présente les caractères suivants :

- des hyphes toutes couchées dont le diamètre est extrêmement petit $(1 \mu$ et même quelquefois moins);

— des articles (cellules uninucléées) de taille variable, mais toujours très longs;

- des « conidiophores» qui ne diffèrent pas des hyphes du mycélium végétatif ;

— des conidies disposées, soit en bouquets, soit au long des hyphes. Ce sont des amérospores qui se présentent, soit sous la forme en pétale de rose, soit sous la forme en ligule de marguerite.

B) Microscopie Électronique a transmission.

L'étude en microscopie électronique à transmission de la forme mycélienne du Sporotrichum schenckii s'est révélée d'un grand intérêt, à cause de la très petite taille des hyphes et des limites imposées par le faible pouvoir séparateur du microscope optique. 
Nos recherches ultrastructurales ont mis en évidence :

- une organisation complexe au niveau des parois et des septa des hyphes, montrant la présence de formations particulières, communes aux Septomycètes et aux Floridées, à savoir, en utilisant la terminologie de $\mathbf{M}$. Chadefaud: une vagina, une sous-vagina, un film doublant intérieurement la sous-vagina, une locula et un appareil interloculaire. Ces structures avaient déjà été observées par M. Chadefaud et par divers auteurs, dans les hyphes d'autres champignons et chez des algues filamenteuses ;

- un appareil synaptique très particulier, formé de deux disques convexes traversés par des trabécules. Cet appareil est très proche de celui des Ascomycètes parfaits et rappelle nettement celui des Floridées;

- la présence de corps de Woronin, dans les articles jeunes et adultes, au voisinage des septa. Dans les articles âgés, ces corps ne sont plus observés;

- un plasmalemme typique, dans les hyphes jeunes et adultes. Dans les hyphes senescentes, il peut être interrompu par endroits. Il peut même former entre le cytoplasme et la paroi cellulaire des profils membranaires, souvent entassées parallèlement les uns aux autres;

- l'existence de nombreux lomasomes. Ces formations, qui évoquent parfois les mésosomes bactériens, pourraient avoir une fonction respiratoire ou une fonction excrétrice ;

- la pauvreté du réticulum endoplasmique;

— de très nombreux ribosomes libres disséminés régulièrement dans le hyaloplasme des articles jeunes. Dans les articles adultes, ils sont souvent groupés en amas au voisinage des vacuoles, qui sont typiques;

- l'absence d'appareil de Golgi ;

- des mitochondries toujours très petites, mais très polymorphes, avec des formes ovoïdes, en haltère ou irrégulières et des formes annulaires remarquables ;

- l'absence de lipides dans les apex des hyphes, car ils n'apparaissent que loin en arrière de leur extrémité distale :

- la présence de particules $\alpha$ et $\beta$ d'un glycogène typique.' Leur ultrastructure est très différente de celle de l'amidon floridéen;

- des noyaux arrondis ou ovoïdes. Dans les hyphes âgées, leur contour peut avoir des échancrures assez importantes.

C) Microscopie électronique a balayage.

Nos observations de la forme mycélienne du Sporotrichum schenckii ont montré :

- des hyphes couchées et des «conidiophores» indifférenciés par rapport au mycélium végétatif. Le Sporotrichum schenckii est donc un Sporotriché;

- mais surtout la présence de figures de copulation: deux hyphes dressées se font face et s'accolent au niveau de leur quart supérieur qui est renflé. Le Sporotrichum schenckii ne peut donc plus être classé parmi les Fungi imperfecti. 


\section{II. - Résultats apportés par nos recherches morphologiques et ultrastructu- rales sur la phase levure du Sporotrichum schenckii.}

A) Microscopie photonique.

$1^{\circ}$ L'étude en microscopie optique de la forme levure du Sporotrichum schenckii cultivé in vitro nous a permis d'observer :

- le polymorphisme cellulaire: dans les cultures jeunes, les levures sont arrondies ou ovoïdes, mais elles peuvent aussi avoir des aspects en cigare, en navette, en pétales de rose ou en ligule de marguerite; dans les cultures âgées, elles peuvent prendre des formes bizarres (formes d'involution);

- le mode de division par bourgeonnement, typiquement unilatéral, mais parfois bilatéral. Il n'y a pas de cloisonnement transversal;

- une morphologie semblable à celle qui existe chez l'hôte in vivo ;

- la présence de rares images évoquant des figures de copulation.

$2^{\circ}$ Dans les lésions histologiques observées chez les animaux inoculés avec une suspension de conidies du Sporotrichum schenckii, nous avons rencontré des éléments parasitaires très polymorphes : corps en cigare ou en navette, amas zoogléiques, figures cercarioïdes, en grains de mil ou sphéroïdes, ou éléments bactérioïdes, et enfin sphérules astéroïdes. Nous n'avons obtenu ces sphérules que chez le hamster et encore difficilement. Les massues des corps astéroïdes ne sont pas d'origine fungique comme en témoigne leur absence de colorabilité par le réactif de Schiff. Elles peuvent être interprétées comme le résultat d'un phénomène de défense de l'hôte. Les massues sont formées d'une substance éosinophile, vraisemblablement élaborée par les tissus de l'hôte.

B) Microscopie électronique a balayage.

L'examen en microscopie à balayage de la forme levure ne nous a apporté aucun élément intéressant.

C) Microscopie électronique a transmission.

Nos observations de la phase levure, avec le microscope électronique à transmission, ont montré :

- l'absence de capsule entourant les levures. Le halo visible autour d'elles en microscopie photonique est donc un artéfact optique;

- l'épaisseur plus grande des parois des levures, comparativement à celle des parois des hyphes: pratiquement le double;

- la faible densité aux électrons de leurs parois. Cependant, nous avons pu y mettre en évidence une mince couche externe ou vagina, une épaisse couche interne qui est peut-être une sous-vagina, plus probablement une locula et un film doublant intérieurement cette couche interne;

- un plasmalemme légèrement ondulé. Dans les levures adultes ou sénescentes, il s'en détache parfois des lames qui s'enfoncent profondément dans le cytoplasme; 
- des lomasomes, beaucoup moins nombreux dans la forme levure que dans la forme mycélienne. Leur rareté est peut-être en relation avec les conditions d'anaérobiose dans laquelle cette phase est cultivée;

- l'absence de réticulum endoplasmique. Ce réticulum était déjà très peu abondant dans la phase mycélienne aérobie. La phase levure étant anaérobie, il est possible que le réticulum disparaisse pendant l'adaptation à ce mode de vie ;

- des ribosomes libres, disséminés dans le hyaloplasme. Nous n'avons jamais observé leur groupement en amas au voisinage des vacuoles comme dans les hyphes;

- l'absence d'appareil de Golgi ;

- des vacuoles beaucoup moins nombreuses dans la phase levure que dans la phase mycélienne. Cette rareté pourrait être due aux conditions de culture de la forme levure en anaérobiose et en milieu glucosé;

- des mitochondries très différentes de celles du mycélium. Leurs modifications morphologiques doivent être en rapport avec l'anaérobiose;

- des noyaux typiques.

Mais surtout, au cours de nos recherches ultrastructurales sur la phase levure, dans les cultures âgées, nous avons découvert des asques et des ascospores. Les asques doivent dériver des couples de filaments sexuels portés par le mycélium et peut-être également des copulations que nous avons observées, mais trop incomplètement, entre des cellules de levures. L'asque mûr est de forme oblongue. Il présente des extrémités amincies. Il mesure $5 \mu$ de long sur $2,5 \mu$ de large. Il renferme quatre ascospores de forme relativement plus allongée, mesurant $3 \mu$ de long sur $1 \mu$ de large. Du fait de la découverte des asques et des ascospores, l'espèce $S p$. schenckii ne peut plus être considérée comme Adélomycète mais doit désormais être rattachée à la classe des Ascomycètes. La présence d'hyphes et de levures, l'absence d'ascocarpe permettent de ranger l'espèce $S p$. schenckii dans la sous-classe des Hémiascomycètes. Le mycélium bien développé, l'absence d'ascocarpe, le mode de formation de l'asque à partir de deux hyphes spéciales rattachent cette espèce à l'ordre des Endomycétales et à la famille des Endomycetaceæ. Dans cette famille, nous n'avons trouvé aucun genre auquel rattacher l'espèce $S p$. schenckii. C'est du genre Eremascus qu'elle se rapproche le plus, mais elle n'en a ni les ascospores globuleuses, ni le mycélium épais. Par son mycélium extrêmement fin, par son appareil de multiplication végétative caractéristique et, surtout, par la forme très allongée de ses asques et de ses ascospores, l'espèce $S p$. schenckii nous a paru devoir constituer le type d'un genre nouveau, pour lequel nous avons proposé le nom de Dolichoascus Thibaut et Ansel 1970.

\section{Conclusion.}

Nos recherches sur le Sporotrichum schenckii (Hetkoen et Perkins 1900) ont montré :

- des caractères d'Ascomycètes au niveau des parois, des septa et de l'appareil synaptique des hyphes;

- mais surtout une forme parfaite de reproduction sexuée, jusqu'alors inconnue. Nos 
travaux dès lors apportent des éléments taxinomiques nouveaux qui changent complètement la place de cette espèce dans la classification des champignons.

L'existence de figures de copulation, d'asques et d'ascospores, ne permet plus de ranger le Sporotrichum schenckii parmi les Fungi imperfecti. Les caractères de sa forme parfaite obligent à le rattacher aux Hémiascomycètes et à la famille des Endomycetaceae. Le Dolichoascus schenckii Thibaut et Ansel 1970 (Hetkoen et Perkins 1900) est donc un Ascomycète, un Hémiascomycète, de l'ordre des Endomycétales et de la famille des Endomycetaceae.

\section{Bibliographie}

Ansel (M.), Thibaut (M.) et Tourte (Y.), 1969. - Les lomasomes du Sporotrichum schenckii. Annales de Parasitologie humaine et comparée, 44, 811-820.

-, 1970. - Une nouvelle Endomycetaceae: Dolichoascus nov. gen. Découverte de la reproduction sexuée par asques chez Sporotrichum schenckii (Hetkoen et Perkins 1900). C.R. Acad. Sc., 270, 2171-2173.

—, 1971. - Réalités et hypothèses sur la forme de reproduction sexuée du Sporothrix schenckii. Annales de Parasitologie humaine et comparée.

Thibaut (M.), 1969. - Les pseudo-synapses dans la forme mycélienne du Sporotrichum schenckii (Hetkoen et Perkins, 1900). Annales de Parasitologie humaine et comparée, 44, 811-820.

-, 1970. - Ultrastructure de la paroi et des septa dans les hyphes du Sporotrichum schenckii (Hetkoen et Perkins 1900). Annales de Parasitologie humaine et comparée, 45, 139-145.

-, 1970. - Biotopologie et dimorphisme du Sporotrichum schenckii (Hetkoen et Perkins 1900). Annales de Parasitologie humaine et comparée, 45, 365-380.

-, 1970. - Etude morphologique de la phase mycélienne du Sporotrichum schenckii (Hetkoen et Perkins 1900) en microscopie photonique. Annales de Parasitologie humaine et comparée, 45, 509-516.

-, 1970. - Recherches sur l'ultrastructure de la forme levure du Sporotrichum schenckii (Hetkoen et Perkins 1900). Annales de Parasitologie humaine et comparée, 46, 677-697.

—, 1970. - Recherches sur l'ultrastructure de la forme mycélienne du Sporotrichum schenckii (Hetkoen et Perkins 1900). Annales de Parasitologie humaine et comparée, 46 699-760.

—, 1970. - Etude biologique, morphologique, cytologique et taxinomique du Sporotrichum schenckii (Hetkoen et Perkins, de Beurmann et Gougerot: Dolichoascus schenckii (H. et P.) Thibaut et Ansel 1970. Thèse de Doctorat ès Sciences, Paris, 1 vol., 233 pages et 47 planches hors-texte.

-, 1970. - Etude morphologique de la phase parasitaire du Sporotrichum schenckii (Hetkoen et Perkins 1900). Annales de Parasitologie humaine et comparée, 45, 881-889.

—, 1971. - Taxinomie, synonymie, historique et répartition géographique du Sporotrichum schenckii (Hetkoen et Perkins 1900). Annales de Parasitologie humaine et comparée, 46, 93-102. 
-, 1971. - Etude morphologique de la phase levure du Sporotrichum schenckii (Hetkoen et Perkins 1900). Annales de Parasitologie humaine et comparée, 46, 81-91.

-, 1971. - Recherches en microscopie à balayage sur la morphologie du Sporotrichum schenckii (Hetkoen et Perkins 1900). Annales de Parasitologie humaine et comparée, 46, 321-328.

—, 1972. - La forme parfaite du Sporotrichum schenckii (Hetkoen et Perkins 1900): Dolichoascus schenckii Thibaut et Ansel 1970 nov. gen. Annales de Parasitologie humaine et comparée, 47, 431-441.

-, Ansel (M.) et LARIVIÈre (M.), 1970. - Aspects cytologiques de quelques éléments du métabolisme chez un Sporotrichum. Annales de Parasitologie humaine et comparée, 45, 497-507. 